\title{
Role of caveolin-1 in chronic postsurgical pain in rats
}

\author{
SAI-SAI HUANG, SU CAO, YI-BIN QIN, CUI E. LU and SHI-REN SHEN \\ Department of Anesthesiology, Affiliated Hospital of Nantong University, Nantong, Jiangsu 226001, P.R. China
}

Received November 24, 2020; Accepted August 17, 2021

DOI: $10.3892 / \mathrm{etm} .2021 .10724$

\begin{abstract}
Chronic postsurgical pain (CPSP) has a high incidence, but the underlying mechanisms remain elusive. Previous studies have indicated that caveolin-1 (Cav-1) plays a notable role in pain modulation. To study the role of Cav-1 in CPSP in the present study, a rat model of skin/muscle incision and retraction (SMIR) was established. Under anesthesia, skin and superficial muscle of the medial thigh were incised and a small pair of retractors inserted. It was revealed that SMIR increased the expression of Cav-1 in the dorsal root ganglion (DRG) and the injured tissue around the incision. Furthermore, the infiltration of endothelial cells and macrophages in the injured tissue around the incision increased constantly, and the vascular permeability increased due to the destruction of the vascular endothelial barrier function around the injured tissue. Cav-1 was mainly expressed by CD68-positive macrophages and CD34-positive endothelial cells in the injured tissues around the incision, while it was also primarily localized in the medium and large neurofilament 200-positive neurons and a small number of calcitonin gene-related peptide- and isolectin B4-positive small and medium-sized neurons in the DRG. The results demonstrated that the sustained high expression levels of Cav-1 in the injured tissue around the incision could lead to the dysfunction of the vascular endothelial barrier and, thus, could induce the inflammatory response through the lipoprotein transport of endothelial cells, thereby resulting in peripheral sensitization. In addition, the sustained high expression levels of Cav-1 in the DRG could sensitize large-sized neurons and change the transmission mode of noxious stimuli. The findings of the present study indicated that a Cav-1-mediated process could participate in neuronal transmission pathways associated with pain modulation.
\end{abstract}

\section{Introduction}

Following surgery, 8-34\% of patients worldwide experience chronic postsurgical pain (CPSP), which leads to a

Correspondence to: Dr Su Cao, Department of Anesthesiology, Affiliated Hospital of Nantong University, 20 Xisi Road, Nantong, Jiangsu 226001, P.R. China

E-mail:mzkcs@sina.com

Key words: caveolin-1, chronic postsurgical pain, dorsal root ganglion, injured tissue around the incision decline in the quality of postoperative daily life $(1,2)$. In the clinic, the therapeutic management for CPSP is limited. In persistent postsurgical pain, there is a series of complicated alterations ranging from nociceptive stimulation to the occurrence and development of postsurgical pain $(3,4)$. It is generally considered that peripheral sensitization is the starting point of CPSP $(5,6)$. Therefore, there is a requirement to discover targets against peripheral sensitization leading to CPSP. Caveolae, which constitute specific forms of lipid rafts, are small invaginations of the plasma membrane that exist in numerous mammalian cells $(7,8)$. Previous studies have reported that caveolae function as organizing centers for signaling molecules $(9,10)$. Caveolins have cytoplasmic $\mathrm{N}$ and $\mathrm{C}$ termini, palmitoylation sites and a scaffolding domain that facilitates interaction and organization of signaling molecules so as to help provide coordinated and efficient signal transduction. Such signaling components include upstream entities (such as $\mathrm{G}$ protein-coupled receptors, receptor tyrosine kinases and steroid hormone receptors) and downstream components (such as heterotrimeric and low-molecular weight $\mathrm{G}$ proteins, effector enzymes and ion channels) (11). Caveolin-1 (Cav-1) is the principal structural and signaling component of caveolae, which is expressed mainly in inflammatory cells $(12,13)$. Recent studies have revealed that Cav-1 plays an important role in pain modulation. For example, in mouse anterior cingulate cortex neurons, Cav-1 contributes to pain modulation by directly binding with $\mathrm{N}$-methyl D-aspartate receptor subtype $2 \mathrm{~B}$, the promotion of which leads to central sensitization $(14,15)$. In diabetic neuropathic pain (DNP) rats, Cav-1 in the spinal cord dorsal horn contributes to the development of DNP through the upregulation of toll-like receptor 4 expression in the spinal cord (16). However, the role of Cav-1 in peripheral sensitization is not clear. It is well known that tissue injury results in endothelial hyperpermeability and increased exudation, which can lead to increased levels of local inflammatory agents that cause sensitization and increased excitation of the nociceptors, thereby causing pain $(17,18)$. Previous studies have demonstrated that there is a positive correlation between Cav-1 overexpression and extravascular albumin, which suggests that upregulation of Cav-1 may be associated with endothelial hyperpermeability (19-21).

In the present study, the skin/muscle incision and retraction (SMIR) model (22) was established to observe the expression and localization of Cav-1 in the tissue around the incision and the dorsal root ganglion (DRG) and examine the effect of Cav-1-induced acute postsurgical pain on chronic pain. 


\section{Materials and methods}

Animals. A total of 84 male Sprague-Dawley rats (weight, 200-250 g; age, 8-10 weeks) were provided with food and water ad libitum. The ratio of light to darkness was $12: 12 \mathrm{~h}$, with the temperature at $23 \pm 1^{\circ} \mathrm{C}$ and a humidity of $55-60 \%$. The rats were provided by The Experimental Animal Center of Nantong University, and the study procedures were approved by the Experimental Animal Protection and Care Committee of Nantong University (approval no. 20171015S1051122; Nantong, China).

SMIR model and injection of drugs. The rats were randomly divided into five groups. In the naive group (12 rats in total), no treatment was performed, six rats were sacrificed and sampled 7 days later. In the sham group (12 rats in total), the rats received an incision through the skin and muscle. In the SMIR group (36 rats in total), after anesthetization under isoflurane induction with $3-4 \%$ induction and 1-2\% maintenance and fixed in the supine position, the rats underwent retraction for $1 \mathrm{~h}$ following the skin/muscle incision. A total of 30 rats in the SMIR group were sacrificed and sampled 1, 3, 7, 14 and 28 days after SMIR modeling with six rats sacrificed at each time point. In the SMIR + Cav-1 small interfering (si)RNA group (12 rats in total), Cav-1 siRNA (Guangzhou RiboBio Co., Ltd.) intrathecal injections were performed at 1, 3 and 7 days after SMIR modeling. For the intrathecal injections, the animals were anesthetized via inhalation of $2 \%$ isoflurane. A spinal cord puncture was made using a 30 -gauge needle between the L4 and L5 level, and $10 \mathrm{nmol} \mathrm{Cav-1} \mathrm{siRNA} \mathrm{was}$ diluted into sterile water containing 5\% dextrose $(40 \mu \mathrm{l})$ to be delivered to the cerebral spinal fluid. Immediately after the needle entry into the subarachnoid space, a brisk tail flick could be observed. In the SMIR + Negative control group (12 rats in total), the control siRNA intrathecal injections were performed at 1, 3 and 7 days after SMIR modeling. Intrathecal injections were performed as aforementioned. Cav-1 siRNA and control siRNA were purchased from Santa Cruz Biotechnology, Inc. The sequence of Cav-1 siRNA was sense, 5'-AACCAGAAGGGACACACAG-3', and antisense, 5'-CUG UGUGUCCCUUCUGGUU-3'. The control siRNA was a non-targeting siRNA, the sequence was sense, 5'-GAGAAG CAGUGAUUACGACG-3', and antisense, 5'-CGUCGUAUC ACUGCUUCUC- 3 '. In the Sham, SMIR + Cav-1 siRNA and SMIR + negative control groups, six rats in each group were sacrificed and sampled 7 days after surgery. SMIR surgery did not cause death or any signs of illness, such as hair loss, diarrhea or weight loss. The rats were sacrificed in a $\mathrm{CO}_{2}$ chamber and the injured tissue around postoperative incision and DRG were collected for subsequent experiments and stored in a $-80^{\circ} \mathrm{C}$ freezer for further use.

Behavioral testing. The absence of heat hyperalgesia has been previously reported with animal models of incisional pain (23). Therefore, the mechanical withdrawal threshold (MWT) was detected prior to and at 1, 3, 7, 14 and 28 days following SMIR surgery in the present study. The rats were habituated to the testing environment for at least $30 \mathrm{~min}$ before testing. Mechanical allodynia was assessed using the up-down paradigm with von Frey filaments (IITC Life Science, Inc.) ranging between 1.4-26 g. Shrinking, swinging or paw licking were regarded as positive reactions. Each filament was presented five times within $30 \mathrm{sec}$ to determine the response threshold. If the response was not elicited at least twice, the next ascending von Frey filament was applied until at least two responses were observed.

Immunofluorescence staining. On day 7 after SMIR, the rats were anesthetized with isoflurane (induction with 3-4\%; maintenance with 1-2\%) and were transcardially perfused with PBS followed by $4 \%$ paraformaldehyde in PBS ( $250 \mathrm{ml} ; \mathrm{pH}$ 7.0). After perfusion, the injured tissues around the postoperative incision and DRG tissues from the rats in the SMIR group were extracted and post-fixed in the $4 \%$ paraformaldehyde at $4^{\circ} \mathrm{C}$ overnight, and then placed in $20 \%$ and subsequently in $30 \%$ sucrose solution at $4^{\circ} \mathrm{C}$ overnight. After embedding with OCT, the tissues were consecutively sectioned at a $6-\mu \mathrm{m}$ thickness and stored at $-20^{\circ} \mathrm{C}$. These sections were selected randomly and blocked with 5\% serum antibody blocking solution (Beyotime Institute of Biotechnology) for $2 \mathrm{~h}$ at room temperature. The tissue slices were then incubated with antibodies against Cav-1 (1:50; cat. no. sc-53564; Santa Cruz Biotechnology, Inc.), CD34 (1:100; cat. no. ab81289; Abcam), CD68 (1:50; cat. no. ab125212; Abcam), calcitonin gene-related peptide (CGRP; 1:800; cat. no. 14959; Cell Signaling Technology, Inc.) and neurofilament 200 (NF200; 1:2,000; cat. no. N5389; Sigma-Aldrich; Merck KGaA) at $4^{\circ} \mathrm{C}$ overnight, then co-incubated with $\mathrm{Cy} 3$-conjugated goat anti-rabbit (1:1,000; cat. no. 111-165-003; Jackson ImmunoResearch Laboratories, Inc.), FITC-conjugated goat anti-mouse secondary antibodies (1:1,000; cat. no. 115-095-205; Jackson ImmunoResearch Laboratories, Inc.) or FITC-conjugated isolectin B4 (IB4; 1:1,000; cat. no. PR-02; Advanced Targeting Systems, Inc.) in the dark for $2 \mathrm{~h}$ at room temperature. Five sections were randomly selected from the injured tissue around the incision and the DRG of each rat. The localizations of Cav-1 in the injured tissue around the incision and the DRG were examined under a fluorescence microscope (Olympus Corporation; magnification, x200) in the dark to capture images, and ImageJ (National Institutes of Health) was used to quantitate fluorescence intensity.

Detection of local tissue vascular permeability. Rats were anesthetized as previously described. Subsequently, $1 \mathrm{ml} / \mathrm{kg}$ of $2 \%$ Evans blue solution was slowly injected into the left femoral vein. If the skin of the toes and ears turned blue, the injection was successful. After $60 \mathrm{~min}$, the rats' chests were opened, and 100-200 $\mathrm{ml}$ of normal saline was perfused from the ascending aorta of the left ventricle, until a clear fluid flowed from auricula dextra. The same portion of the injured tissue around postoperative incision was collected and placed in an Eppendorf tube for use. The 2\% Evans blue solution was diluted 100 -fold, and a concentration of $20 \mathrm{ng} / \mu \mathrm{l}$ of the liquid was serially diluted into $10,5,2.5,1.25,0.625,0.313$ and $0.156 \mathrm{ng} / \mu \mathrm{l}$, using formamide as blank, in order to make an Evans blue standard curve. The tissue was placed in a $2 \%$ carboxamide bath at a ratio of $100 \mathrm{mg}: 1 \mathrm{ml}$, homogenized, incubated at $37^{\circ} \mathrm{C}$ for $48 \mathrm{~h}$ and then centrifuged at high speed $(13,527.8 \times \mathrm{g})$ at $4^{\circ} \mathrm{C}$ for $15 \mathrm{~min}$, the supernatant of which was carefully aspirated and divided into three sample tubes 
(50 $\mu \mathrm{l} /$ tube). The absorbance was measured at $620 \mathrm{~nm}$ using a microplate reader (BioTek Instruments, Inc.). According to the standard curve, the Evans blue content of the sample was calculated, and the mean value was recorded to detect the leakage outside the blood vessel. The unit was ng Evans blue/mg tissue.

Western blotting. The rats were anesthetized and sacrificed as previously described, and the injured tissue around the incision and the DRG was homogenized in sodium dodecyl sulfate (SDS; cat. no. 71736; Sigma-Aldrich; Merck KGaA) sample buffer containing a mixture of protease and phosphatase inhibitors (Sigma-Aldrich; Merck KGaA), and measured with a BCA protein assay kit (Beyotime Institute of Biotechnology). For separation, $30 \mu \mathrm{g}$ total protein per gel lane was loaded onto $10 \%$ gels (Beyotime Institute of Biotechnology). The separated proteins were then transferred onto nitrocellulose membranes. The membranes were incubated for $2 \mathrm{~h}$ at room temperature in TBS $+0.1 \%$ Tween-20 (TBST) blocking solution containing $5 \%$ skimmed milk, followed by overnight incubation at $4^{\circ} \mathrm{C}$ in blocking solution containing primary antibodies against Cav-1 (1:50; cat. no. sc-53564; Santa Cruz Biotechnology, Inc.) and GAPDH (1:5,000; cat. no. SAB2108668; Sigma-Aldrich; Merck KGaA). Membranes were washed three times with TBST (10 min/wash) and incubated with goat anti-mouse (cat. no. 115-035-003) and anti-rabbit (cat. no. 111-005-003) HRP-conjugated secondary antibodies (both 1:2,000; both from Jackson ImmunoResearch Laboratories, Inc.) at room temperature for $2 \mathrm{~h}$. Following washing with TBST, immunolabeling was detected using the Tanon 2500 gel imaging system (Tanon Science and Technology Co., Ltd.) and hypersensitive ECL chemiluminescence detection kit (Absin Bioscience, Inc.). ImageJ software (v1.8.0; National Institutes of Health) was used to capture images and analyze the intensity of the bands.

Reverse transcription-quantitative (RT-q)PCR. Cav-1 mRNA expression levels in the injured tissue around postoperative incision and the DRG were determined via RT-qPCR. Total RNA was extracted from tissue using TRIzol ${ }^{\circledR}$ reagent (cat. no. 15596-026; Invitrogen; Thermo Fisher Scientific, Inc.) according to the manufacturer's instructions. cDNA was synthesized using a RevertAid RT Reverse Transcription kit (cat. no. K1691; Thermo Fisher Scientific, Inc.) according to the manufacturer's instructions. RT-PCR was performed on cDNA using LightCycler 96 Real-Time PCR System with UltraSYBR ${ }^{\circledR}$ Mixture (with ROX) (CoWin Biosciences). Each sample was configured to a $25 \mu 1$ reaction volume, and GAPDH was used as reference gene. Primer sequences were as follows: Cav-1 Forward, 5'-ACCTCAACGATGACGTGG TCAAGA-3', and reverse, 5'-TGGAATAGACACGGCTGA TGCACT-3'; GAPDH forward, 5'-GAAGATGGTGATGGG ATTTC-3', and reverse, 5'-GAAGGTGAAGGTCGGAGTC-3'. Reaction conditions were as follows: Pre-denaturation at $95^{\circ} \mathrm{C}$ for $10 \mathrm{~min}$, followed by 45 cycles of denaturation at $95^{\circ} \mathrm{C}$ for $30 \mathrm{sec}$, annealing at $52^{\circ} \mathrm{C}$ for $40 \mathrm{sec}$ and extension at $72^{\circ} \mathrm{C}$ for $40 \mathrm{sec}$. To ensure primer specificity, a melting curve was set after the 45 amplification cycles $\left(95^{\circ} \mathrm{C}\right.$ for $5 \mathrm{sec}, 65^{\circ} \mathrm{C}$ for $60 \mathrm{sec}$ and $97^{\circ} \mathrm{C}$ for $1 \mathrm{sec}$ ). Results were presented as the level of mRNA relative to endogenous and calculated using the $2^{-\Delta \Delta \mathrm{Cq}}$ method (24).
ELISA. Concentrations of IL-6 (PicoKine ELISA kit; cat. no. EK0411) and TNF- $\alpha$ (PicoKine ELISA kit; cat. no. EK0527) were measured using respective specific ELISA kits in accordance with the manufacturer's instructions (all supplied from Boster Biological Technology).

Data and statistical analyses. Experimental data were analyzed using SPSS 20.0 software (IBM Corp.). Measured data were expressed as the mean \pm SEM, and behavioral data were analyzed by a mixed two-way ANOVA followed by the Bonferroni test for multiple comparison analysis. One-way ANOVA followed by the Bonferroni test was used for comparison between the groups except for the behavioral data. $\mathrm{P}<0.05$ was considered to indicate a statistically significant difference.

\section{Results}

SMIR induces persistent mechanical allodynia and continuous increase of vascular endothelial permeability around the incision. To study the mechanism of postoperative chronic pain, a SMIR model was established. Significant levels of mechanical allodynia appeared on day 1 in SMIR rats and persisted until day 14 after SMIR. MWT in SMIR rats returned to similar levels as in the sham and naive rats by postsurgical day 28 (Fig. 1A).

The tissues around the incision were collected on the 1st, 3rd, 7th, 14th and 28th day after SMIR to detect vascular endothelial permeability using Evans blue staining. It was revealed that surgical wounds significantly induced maintenance of endothelial hyperpermeability in the SMIR group from day 1 after surgery, while compared with the naïve group there was no significant change in the vascular endothelial permeability of the sham group (Figs. 1B and S1; Table SI).

Effects of CPSP on the expression of Cav-1 in the injured tissue around postoperative incision and the DRG. To explore the role of Cav-1 in CPSP and its possible mechanism, changes in the expression of Cav-1 in the injured tissue around postoperative incision and the DRG were examined on postsurgical days 3, 7 and 14 in the SMIR and sham groups. Western blotting demonstrated that compared with the naive and sham groups, the expression of Cav-1 was significantly increased on day 7 and 14 following SMIR in the injured tissue around postoperative incision, while there was no significant change on postsurgical day 3 (Fig. 2A). The expression of Cav-1 was significantly increased on postsurgical day 3 and 7 in the DRG, while there was no significant change on postsurgical day 14 (Fig. 2B). In addition, compared with the naïve group, there was no significant change in the expression of Cav-1 in the injured tissue around postoperative incision and the DRG in the sham group (Fig. 2A and B).

Immunofluorescence staining demonstrates localization of Cav-1 in the injured tissue around postoperative incision. To study the localization of Cav-1, the tissue sections around the postoperative incision of rats in the SMIR group at postsurgical day 7 were stained with the macrophage marker CD68 and the endothelial cell marker CD34. Immunofluorescence staining indicated that Cav-1 was expressed by macrophages 
A

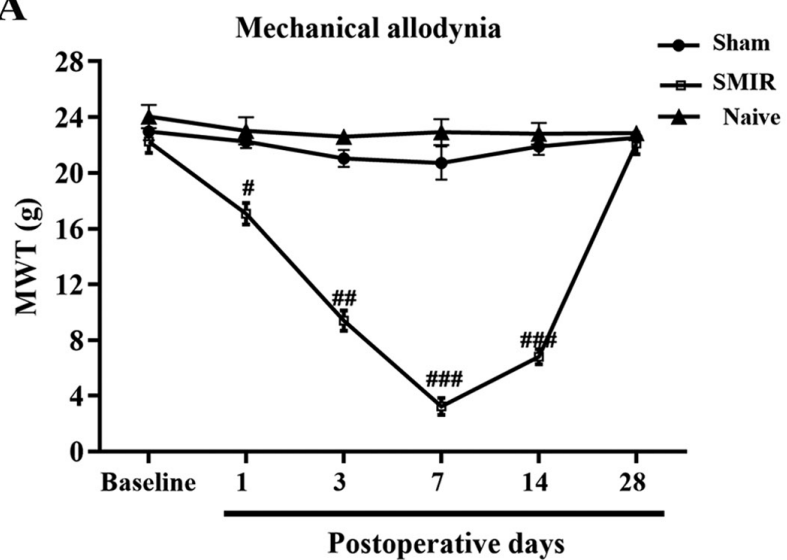

B

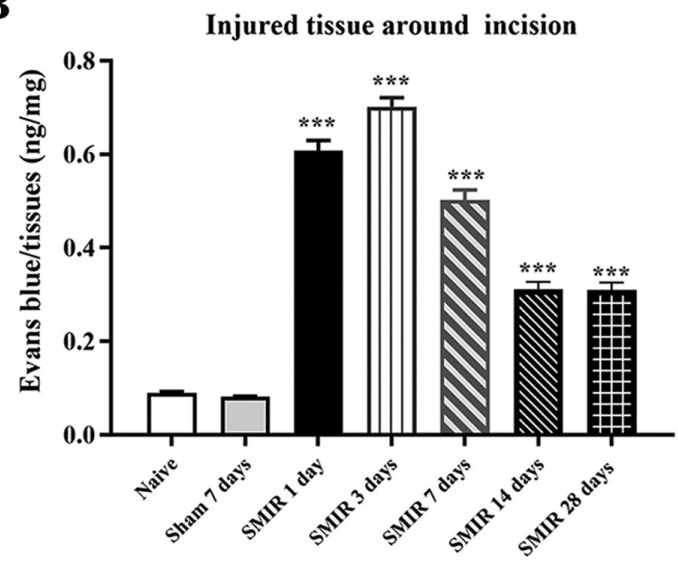

Figure 1. Detection of MWT after SMIR and the vascular endothelial permeability around the incision. (A) MWT of the SMIR group was significantly reduced in a time-dependent manner on days 1,3,7 and 14 after surgery increased after postsurgical day 14, whereas no statistical difference was observed in the sham group compared with the naive group. (B) Intravascular Evans blue extravasation of the SMIR group was significantly increased on days 1, 3, 7, 14 and 28 after surgery. $n=6 .{ }^{\#} \mathrm{P}<0.05,{ }^{\# \#} \mathrm{P}<0.01$ and ${ }^{\# \# \#} \mathrm{P}<0.001$ vs. sham group; ${ }^{* * *} \mathrm{P}<0.001$ vs. naive and sham groups. SMIR, skin/muscle incision and retraction; MWT, mechanical withdrawal threshold; d, day.

A

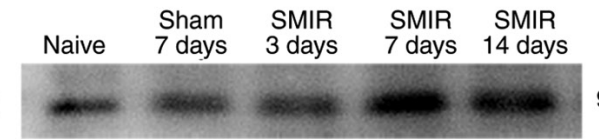

$90 \mathrm{kDa}$

GAPDH

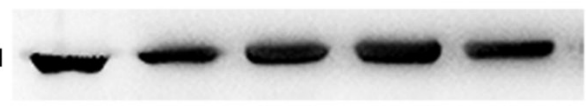

$38 \mathrm{kDa}$

Injured tissue around incision

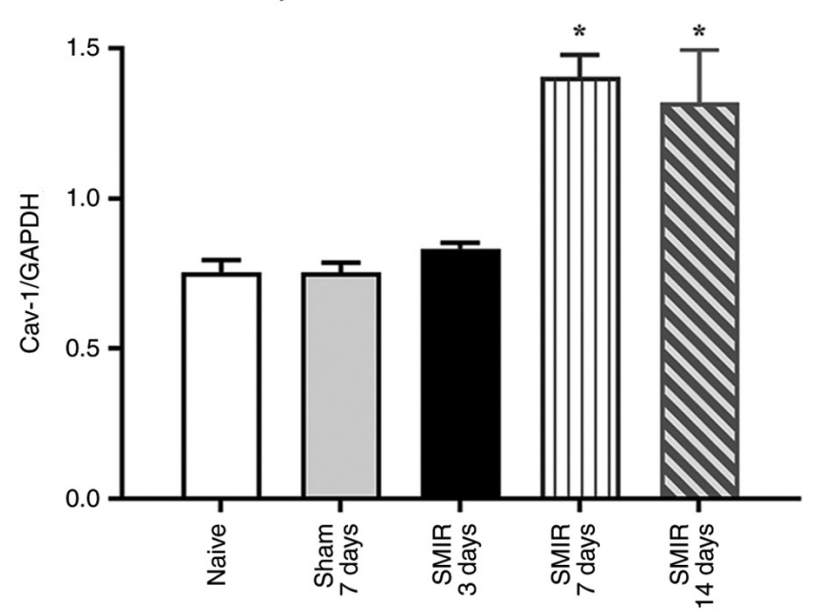

B

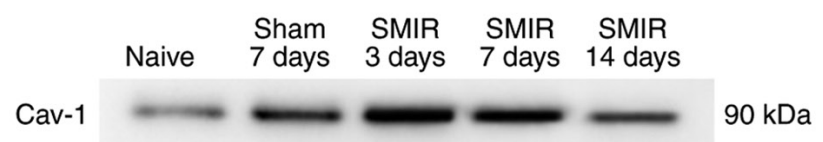

GAPDH

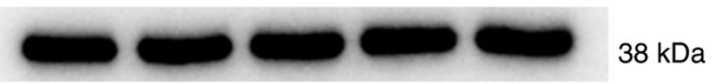

DRG

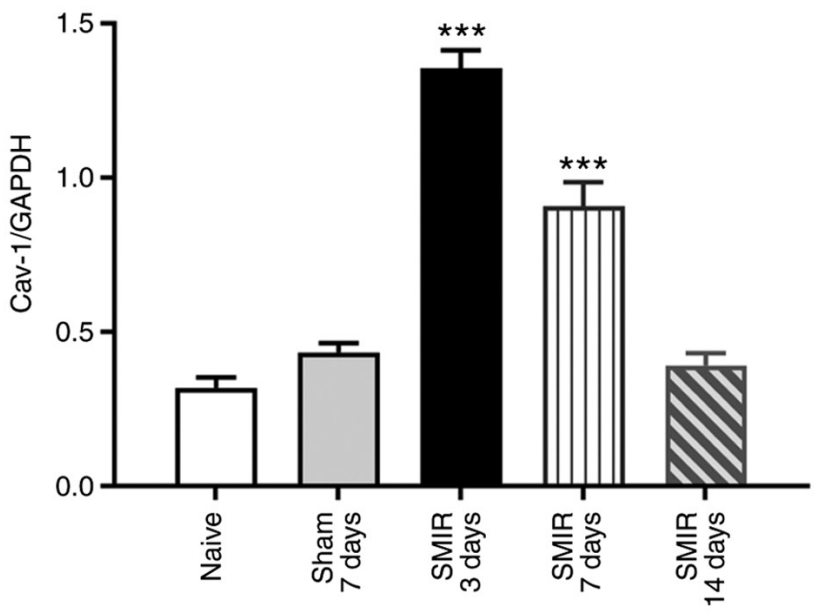

Figure 2. CPSP increases the expression of Cav-1 in the injured tissue around the postoperative incision and DRG. (A) CPSP significantly increased Cav-1 expression in the injured tissue around the incision on day 7 and 14 after surgery. (B) CPSP significantly increased Cav-1 expression in the DRG on day 3 and 7 after surgery. $n=6 .{ }^{*} \mathrm{P}<0.05$ and ${ }^{* * * *} \mathrm{P}<0.001$ vs. the naive and sham groups. Cav-1, caveolin-1; CPSP, chronic postsurgical pain; DRG, dorsal root ganglion; SMIR, skin/muscle incision and retraction.

and endothelial cells in the injured tissue around postoperative incision (Fig. 3).

Immunofluorescence staining demonstrates localization of Cav-1 in the DRG. At postsurgical day 7, DRG tissue sections were stained with Cav-1, the medium and large neuronal marker NF200 or the medium and small neuronal markers CGRP and IB4. The results revealed that Cav-1 was mainly distributed in the NF200-positive medium and large neurons, and a small part of it was distributed in CGRP- or IB4-positive small and medium-sized neurons in the DRG (Fig. 4)

Intrathecal injection of Cav-1 siRNA attenuates mechanical allodynia and decreases Cav-1 mRNA and protein expression in the injured tissue around postoperative incision and the DRG. To further investigate the role of Cav-1 in CPSP, Cav-1 siRNA was used to silence Cav-1 gene expression at 1, 3 and 7 days following SMIR. Compared 

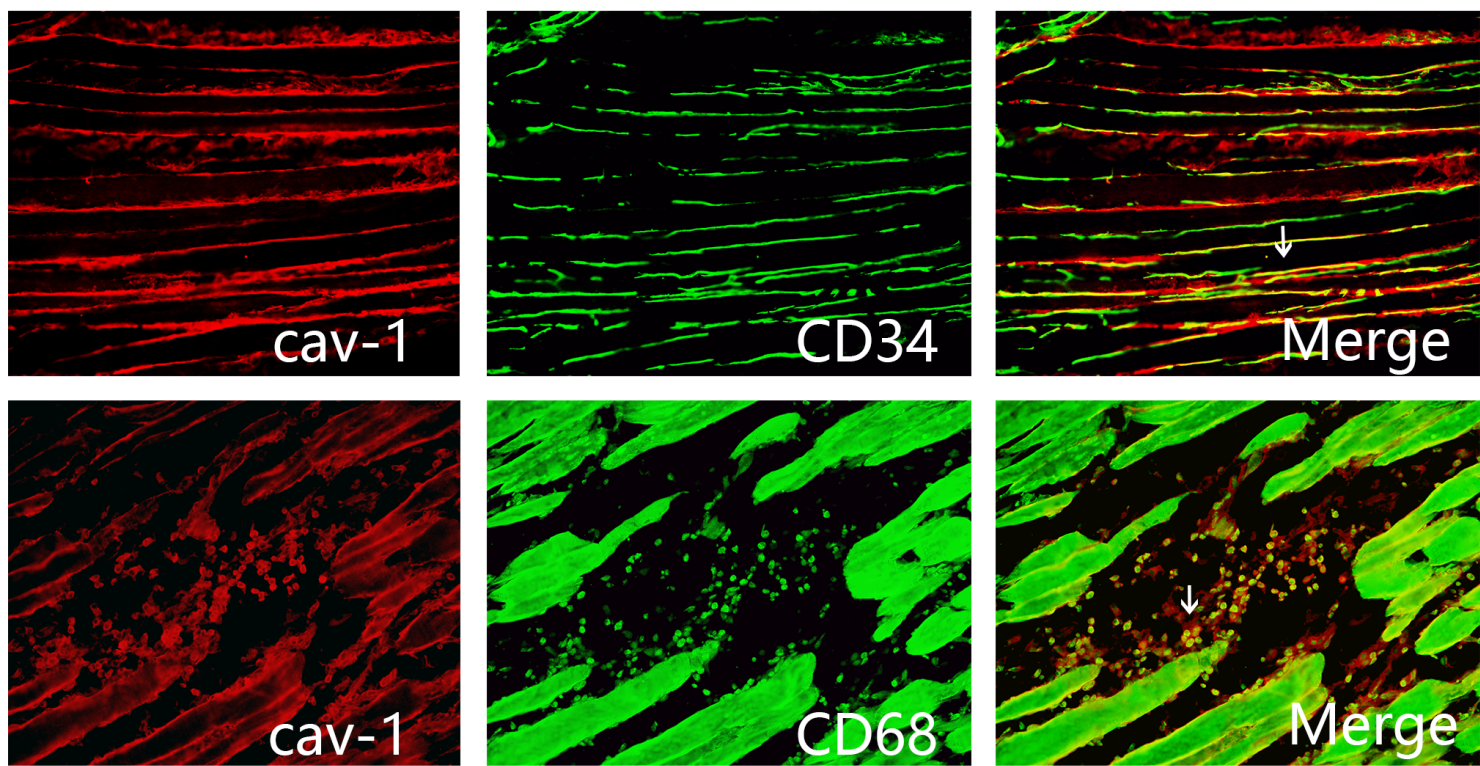

Figure 3. Immunofluorescence staining indicates the localization of Cav-1 in the injured tissue around the incision. Cav-1 was co-localized with the macrophage marker CD68 and the endothelial cell marker CD34 (magnification, x200). The white arrows indicate the position of positive cells. Cav-1, caveolin-1.
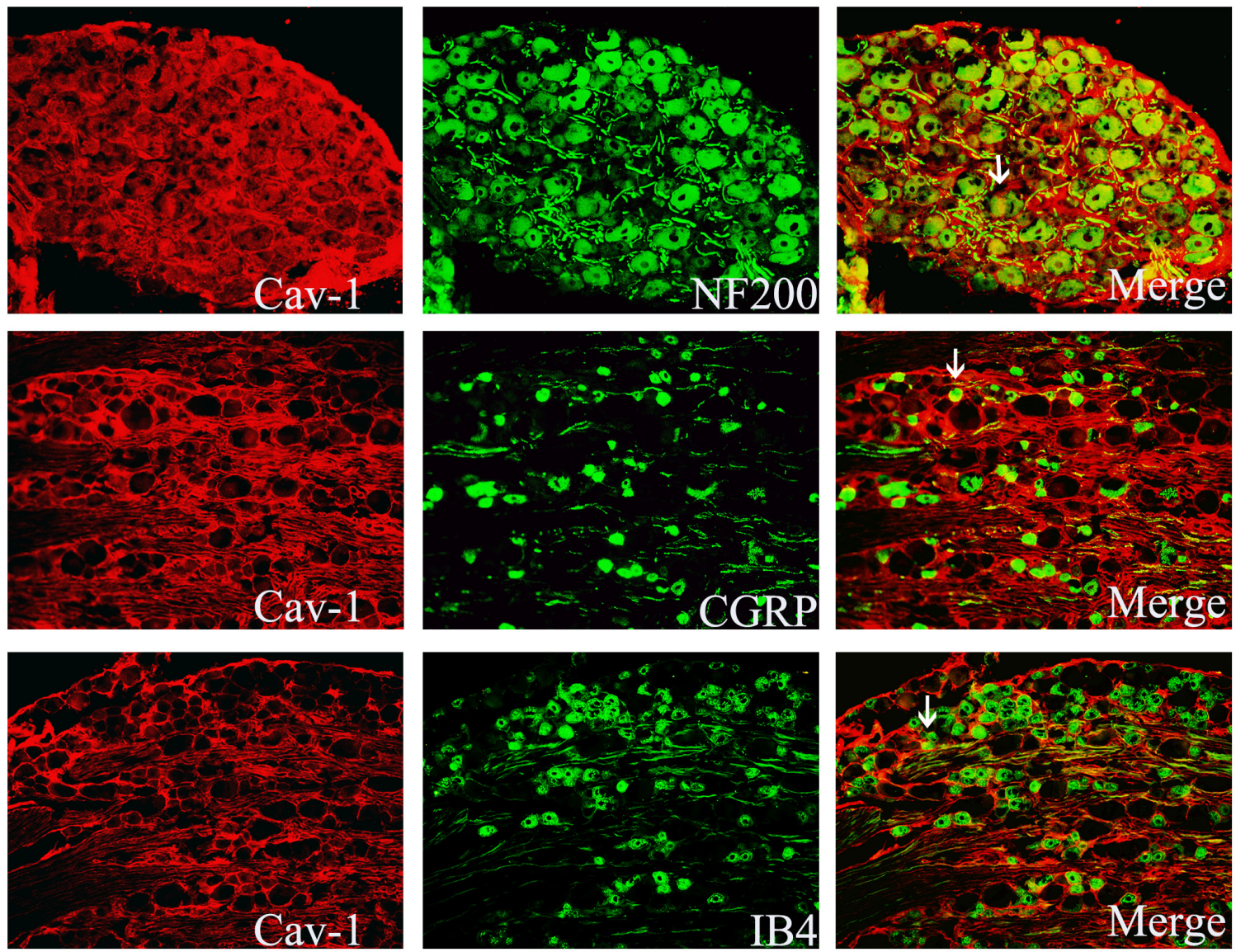

Figure 4. Immunofluorescence staining demonstrates the localization of Cav-1 in the dorsal root ganglion. Cav-1 was mainly distributed in NF200-positive medium and large neurons and only a small part of it was distributed in the CGRP- and IB4-positive medium and small neurons (magnification, x200). The white arrows indicate the position of positive cells. Cav-1, caveolin-1; CPSP, chronic postsurgical pain; NF200, neurofilament 200; CGRP, calcitonin gene-related peptide; IB4, isolectin B4.

with the SMIR + Negative control group, intrathecal injection of Cav-1 siRNA significantly increased MWT on postsurgical days 1, 3, 7 and 14 (Fig. 5A). As presented in
Fig. 5B and C, the expression of Cav-1 in the Cav-1 siRNA group significantly decreased in the injured tissue around postoperative incision and the DRG, as compared with the 
A

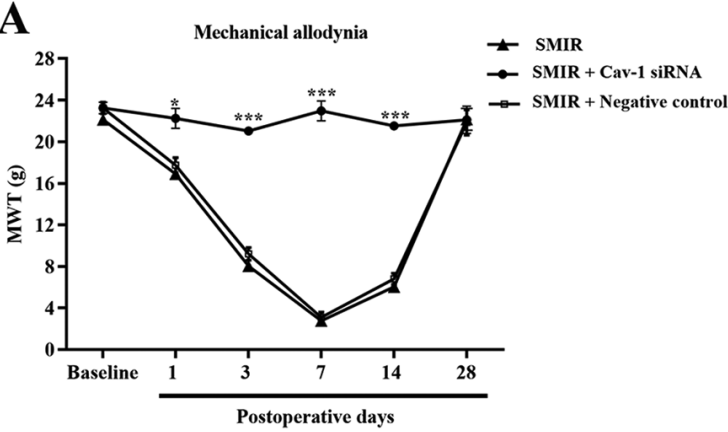

C
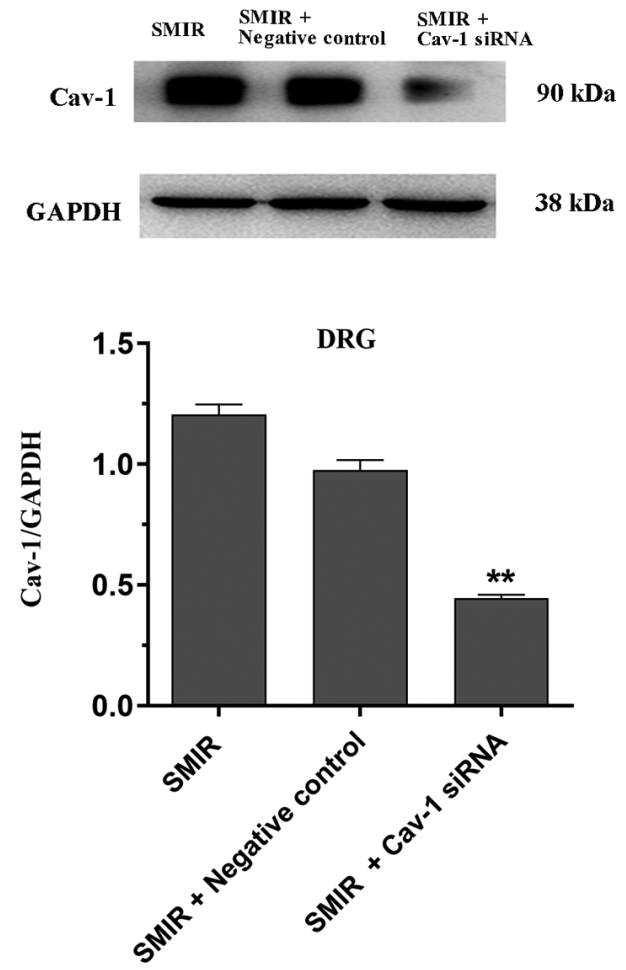

B
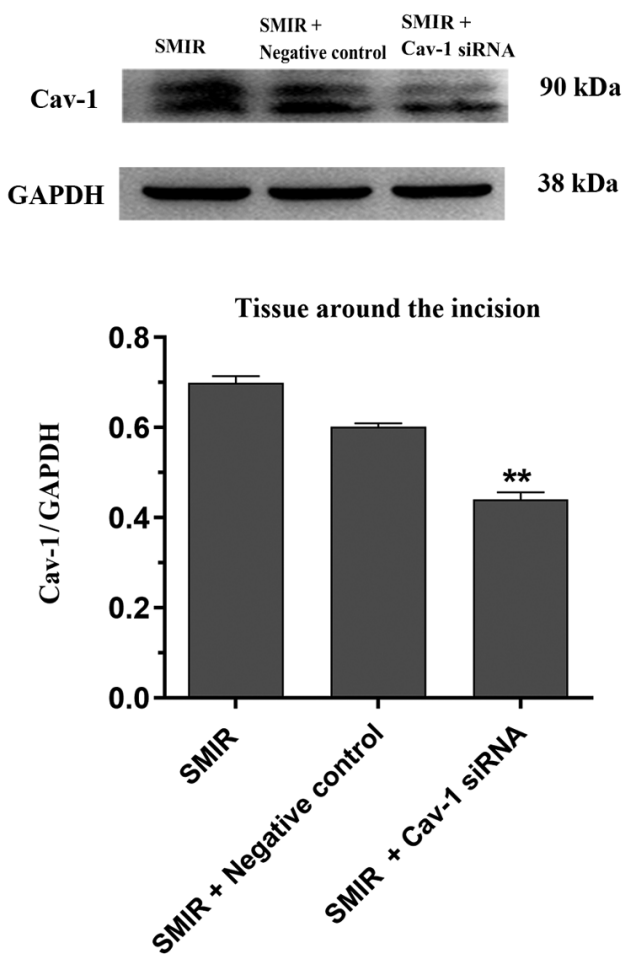

$\mathrm{D}$

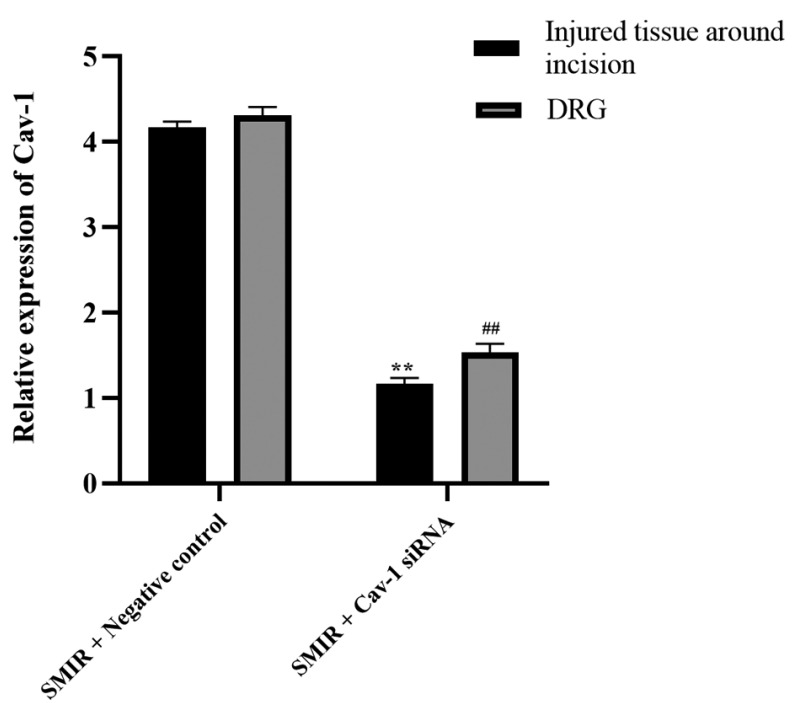

Figure 5. Cav-1 siRNA attenuates mechanical allodynia and decreases Cav-1 mRNA and protein expression in the injured tissue around the postoperative incision and the DRG. (A) Cav-1 siRNA attenuated the SMIR-induced mechanical allodynia on days 1, 3, 7 and 14 after surgery. Cav-1 siRNA decreased the expression levels of Cav-1 in (B) the injured tissue around the postoperative incision and (C) the DRG. (D) Cav-1 siRNA decreased the expression levels of Cav-1 mRNA in the injured tissue around the incision and the DRG compared with the SMIR + Negative control group. $\mathrm{n}=6$. ${ }^{*} \mathrm{P}<0.05$, ${ }^{* *} \mathrm{P}<0.01$ and ${ }^{* * * *} \mathrm{P}<0.001$ vs. SMIR + Negative control group; ${ }^{\# \#} \mathrm{P}<0.01$ vs. the SMIR + Negative control group in the DRG. Cav-1, caveolin-1; si-, small interfering; SMIR, skin/muscle incision and retraction; DRG, dorsal root ganglion; MWT, mechanical withdrawal threshold.

SMIR + Negative control group. RT-qPCR was performed to examine the mRNA levels of Cav-1 in the injured tissue around the postoperative incision and the DRG after intrathecal injection of Cav-1 siRNA. As presented in Fig. 5D, Cav-1 siRNA significantly decreased the expression levels of Cav-1 mRNA in the injured tissue around the incision and the DRG compared with the negative control on postsurgical day 7, reversing the increase in the expression levels of Cav-1 associated with SMIR.
Effects of CPSP on the expression of IL-6 and TNF- $\alpha$ protein levels in the injured tissue around postoperative incision and the DRG. The IL-6 and TNF- $\alpha$ protein levels were detected in the injured tissue around postoperative incision and the DRG following SMIR surgery. ELISA assays demonstrated that IL-6 and TNF- $\alpha$ expression levels were significantly increased in the injured tissue around postoperative incision and the DRG on postsurgical days 3,7 and 14 compared with the naive and sham groups (Fig. 6A and B). 

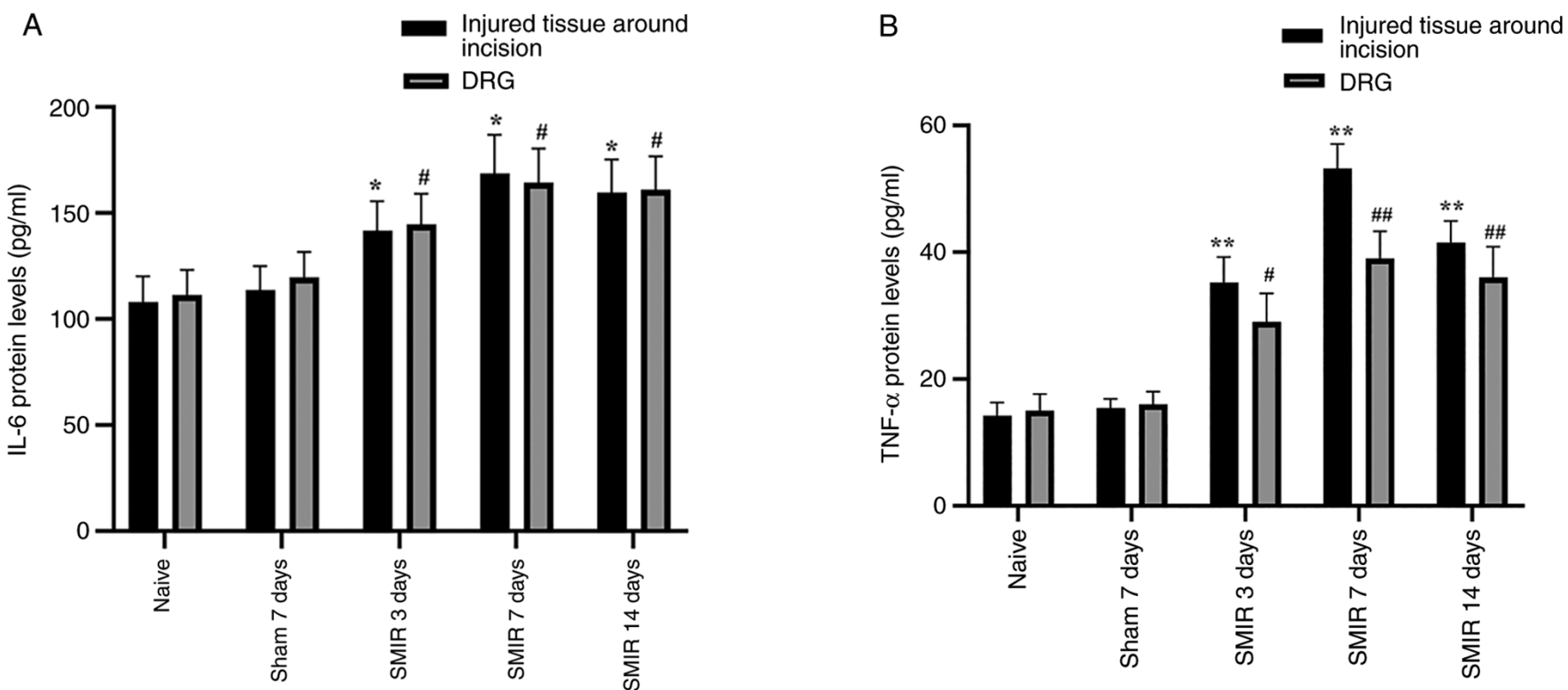

Figure 6. CPSP increases the expression of IL-6 and TNF- $\alpha$ protein levels in the injured tissue around the postoperative incision and DRG. CPSP increased (A) IL-6 and (B) TNF- $\alpha$ expression in the injured tissue around the incision and the DRG on postsurgical days 3 , 7 and 14 compared with the naive and sham groups. $\mathrm{n}=6$. ${ }^{*} \mathrm{P}<0.05$ and ${ }^{* *} \mathrm{P}<0.01$ vs. naïve and sham groups of the injured tissue around the incision; ${ }^{*} \mathrm{P}<0.05$ and ${ }^{\# \#} \mathrm{P}<0.01$ vs. naïve and sham groups of the DRG. DRG, dorsal root ganglion; SMIR, skin/muscle incision and retraction; CPSP, chronic postsurgical pain.

\section{Discussion}

Peripheral sensitization serves an important role in the occurrence and maintenance of CPSP $(25,26)$. The purpose of the present study was to explore the role of Cav-1 in peripheral sensitization, starting with the alterations of the peripheral incision tissue. The caveolae are a special form of lipid raft, which is a small invagination of the plasma membrane that is present in a number of mammalian cells, such as endothelial cells, adipocytes and skeletal muscle $(27,28)$. Previous studies have reported that caveolae are the cellular center of signaling molecules $(9,10,29)$. The formation and stability of the caveolae mainly depends on caveolin (30). Cav-1 is the main structural and signaling component of the fossa, which is mainly expressed in inflammatory cells (31-33). Recent studies have demonstrated that Cav-1 serves a notable role in pain modulation $(14,15)$, but its specific mechanism of action requires further investigation. Our previous study has demonstrated that SMIR increased the infiltration of macrophages and endothelial cells in the injured tissue around the postoperative incision (6), suggesting that the inflammatory response in the tissue around the incision was aggravated, which led to the occurrence of CPSP. In the current study, the results indicated that SMIR increased the permeability of vascular endothelial cells in the muscle tissue around the incision at an early stage (on postoperative day 1). In addition, the expression of Cav-1 increased following SMIR and was localized in macrophages and endothelial cells, suggesting that Cav-1 may be involved in the inflammatory response of the tissue around the incision.

Endothelial cells regulate leukocyte activity, inflammation and platelet aggregation by secreting various active substances, while endothelial hyperpermeability induces inflammatory response in local tissues (34). Due to the minor trauma in the sham operation group, the vascular permeability of the local tissues did not significantly change, therefore there was less local inflammatory exudation accompanied by Cav- 1 expression in the local tissues and the DRG. The results of the present study indicated that Cav-1 was upregulated after the initiation of the inflammatory response in the injured tissue around the postoperative incision. Moreover, a positive correlation between Cav-1 upregulation and the levels of extravascular albumin has been demonstrated, which suggested that upregulation of Cav-1 may be associated with endothelial hyperpermeability $(35,36)$.

It was hypothesized that SMIR increased the expression level of Cav-1 in the tissue around the incision, which resulted in an increase in the permeability of vascular endothelial cells in the tissue. The increase of vascular endothelial permeability then led to a continuous and significant increase in exudation, which aggravated the inflammatory response of the tissue around the incision. This promoted the transmission of pain information to the higher center and, therefore, resulted in CPSP. The pain signals are transmitted along the DRGs to the spinal cord and then to the corresponding zone of the brain, including the thalamus, forebrain, brainstem and midbrain, and finally to the cerebral cortex (37-39). Therefore, as the soma assembly of the first-order neurons for the pain pathway, the excitability of DRGs is important in pain signaling (37-39).

As reported in the literature, early nociceptive stimuli may induce vigorous production of cytokines, such as IL-6 and TNF- $\alpha$, in the DRG, and the cytokines may be transported to central terminals of primary afferents $(40,41)$. Furthermore, the cytokines further activate glial cells and neurons to release more activating substances, such as ATP, pro-inflammatory factors and reactive oxygen species (42). These activating substances further enhance pain and may transform acute pain into chronic pain (43). In the present 
study, the expression levels of the inflammatory factors IL-6 and TNF- $\alpha$ not only increased in the DRG, but also in the tissues surrounding incision after noxious injury, which provided evidence towards the aforementioned hypothesis. In addition, the results of the present study demonstrated that the increase of Cav-1 in the DRG occurred in the SMIR group on the 3rd day after operation, while the increase of Cav-1 in the peripheral tissue was relatively delayed until postoperative day 7; therefore, the early nociceptive stimuli may stimulate the alteration of Cav-1 expression levels in the DRG first.

A previous study has revealed that Cav-1 is present at excitatory synapses and concentrates at the postsynaptic density during the later stage development of presynaptic components (44), suggesting that Cav-1 may play a notable role in synapse formation and plasticity. The results of the present study indicated that Cav-1 was mainly distributed in NF200-positive large and medium neurons in the DRG. In addition, the increased expression of Cav-1 in the DRG after SMIR was associated with a decreased MWT of rats with SMIR. Previous studies have demonstrated that large-sized neurons in the DRG are connected with A- $\beta$ fibers, which transmit the mechanical stimuli $(45,46)$. Central fibers of large-sized neurons in the DRG mainly project to the deep layers of the spinal cord, which is involved in mechanical allodynia $(47,48)$. Therefore, it is possible that in the models of the present study, high expression of Cav-1 in large-sized neurons of the DRG after SMIR sensitized the neurons and changed the transmission mode of noxious stimuli, resulting in mechanical pain hypersensitivity.

To further confirm whether the increase of Cav-1 was involved in pain modulation in rats with CPSP, Cav-1 siRNA was used to silence Cav-1 gene expression. The results indicated that the intrathecal delivery of Cav-1 siRNA decreased the expression levels of Cav-1 mRNA and protein in the tissue around the incision and in the DRG. Furthermore, it significantly prevented the development of hyperalgesia in rats. These results further indicated the association between Cav-1 and CPSP.

The present study is limited in that, except for using Evans blue staining, the expression profiles of intercellular adhesion molecule 1 or vascular cell adhesion protein 1 were not detected to provide evidence of alteration in the vascular permeability.

In summary, peripheral noxious stimuli or injury stimulated Cav-1 expression in the tissue around the incision and in the DRG. The high expression of Cav-1 in the tissue around the incision was accompanied by high permeability of endothelial cells, which modeled the local chronic, inflammatory and nutrient rich microenvironment at the beginning of nociceptive information transmission, thus transmitting the abnormal pain signal to neurons of the DRG. The high expression levels of Cav-1 sensitized large neurons in the DRG and changed the transmission mode of harmful stimuli, which resulted in mechanical pain hypersensitivity. Cav-1 may represent the key link and initial event of peripheral sensitization of CPSP. Targeted Cav-1 intervention may be a potential therapeutic strategy to inhibit peripheral sensitization and provide novel ideas for the treatment of CPSP.

\section{Acknowledgements}

Not applicable.

\section{Funding}

The present study was supported by The National Natural Science Foundation of China (grant no. 81701106).

\section{Availability of data and materials}

All data generated or analyzed during this study are included in this published article.

\section{Authors' contributions}

SSH and SC designed the study. SSH, SRS and CEL acquired and interpreted the data. YBQ, SRS and CEL analyzed the data and assisted SSH in revising the manuscript. SSH and SC prepared the manuscript and supervised the study. SSH and SC confirm the authenticity of all the raw data. All authors have read and approved the final manuscript.

\section{Ethics approval and consent to participate}

All experiments in the current study were approved by The Experimental Animal Protection and Care Committee of Nantong University (approval no. 20171015S1051122; Nantong, China).

\section{Patient consent for publication}

Not applicable.

\section{Competing interests}

The authors declare that they have no competing interests.

\section{References}

1. Kim DH,Pearson-Chauhan KM,McCarthy RJ and Buvanendran A: Predictive factors for developing chronic pain after total knee arthroplasty. J Arthroplasty 33: 3372-3378, 2018.

2. Maguire J, Thibodeau ML and Oliver J: CPSP 2014 results: What have we learned? Paediatr Child Health 20: 435-436, 2015.

3. Uemoto Y, Uchida M, Kondo N, Wanifuchi-Endo Y, Fujita T, Asano T, Hisada T, Nishikawa S, Katagiri Y, Terada M, et al: Predictive factors for patients who need treatment for chronic post-surgical pain (CPSP) after breast cancer surgery. Breast Cancer 22: doi: 10.1007, 2021.

4. Blichfeldt-Eckhardt MR: From acute to chronic postsurgical pain: The significance of the acute pain response. Dan Med J 65: B5326, 2018.

5. Fregoso G, Wang A, Tseng K and Wang J: Transition from acute to chronic pain: Evaluating risk for chronic postsurgical pain. Pain Physician 22: 479-488, 2019.

6. Pan P, Huang SS, Shen SR, Lu CE, Qin YB, Zhang JL and Cao S: Role of p120 catenin in Epac1-induced chronic postsurgical pain in rats. Pain Res Manag 2019: 9017931, 2019.

7. Parton RG, McMahon KA and Wu Y: Caveolae: Formation, dynamics, and function. Curr Opin Cell Biol 65: 8-16, 2020.

8. Tiruppathi C, Regmi SC, Wang DM, Mo GCH, Toth PT, Vogel SM, Stan RV, Henkemeyer M, Minshall RD, Rehman J and Malik AB: EphB1 interaction with caveolin-1 in endothelial cells modulates caveolae biogenesis. Mol Biol Cell 31: 1167-1182, 2020. 
9. Huang $\mathrm{Q}$, Zhong $\mathrm{W}, \mathrm{Hu} \mathrm{Z}$ and Tang $\mathrm{X}$ : A review of the role of cav-1 in neuropathology and neural recovery after ischemic stroke. J Neuroinflammation 15: 348, 2018.

10. de Souza GM, de Albuquerque Borborema ME, de Lucena TMC, da Silva Santos AF, de Lima BR, de Oliveira DC and de Azevêdo Silva J: Caveolin-1 (CAV-1) up regulation in metabolic syndrome: All roads leading to the same end. Mol Biol Rep 47: 9245-9250, 2020.

11. Patel HH, Murray F and Insel PA: Caveolae as organizers of pharmacologically relevant signal transduction molecules. Annu Rev Pharmacol Toxicol 48: 359-391, 2008.

12. Codrici E, Albulescu L, Popescu ID, Mihai S, Enciu AM, Albulescu R, Tanase C and Hinescu ME: Caveolin-1-knockout mouse as a model of inflammatory diseases. J Immunol Res 29: 2498576, 2018

13. Zhang Y, Luo H, Lv X, Liu J, Chen X, Li Y, Liu A and Jiang Y: Axin-1 binds to caveolin-1 to regulate the LPS-induced inflammatory response in AT-I cells. Biochem Biophys Res Commun 513: 261-268, 2019

14. Yang JX, Hua L, Li YQ, Jiang YY, Han D, Liu H, Tang QQ, Yang XN, Yin C, Hao LY, et al: Caveolin-1 in the anterior cingulate cortex modulates chronic neuropathic pain via regulation of NMDA receptor 2B subunit. J Neurosci 35: 36-52, 2015.

15. Chen JL, Lu JH, Xie CS, Shen YJ, Wang JW, Ye XY, Zhang MB Jia GL, Tao YX, Li J and Cao H: Caveolin-1 in spinal cord modulates type-2 diabetic neuropathic pain through the Rac1/NOX2/NR2B signaling pathway. Am J Transl Res 12: 1714-1727, 2020

16. Jia GL, Huang Q, Cao YN, Xie CS, Shen YJ, Chen JL, Lu JH, Zhang MB, Li J, Tao YX, and Cao H: Cav-1 participates in the development of diabetic neuropathy pain through the TLR4 signaling pathway. J Cell Physiol 235: 2060-2070, 2020.

17. Wang Y, Gao Y, Tian Q, Deng Q, Wang Y, Zhou T, Liu Q, Mei K, Wang Y, Liu H, et al: TRPV1 SUMOylation regulates nociceptive signaling in models of inflammatory pain. Nat Commun 9: $1529,2018$.

18. Bang S, Xie YK, Zhang ZJ, Wang Z, Xu ZZ and Ji RR: GPR37 regulates macrophage phagocytosis and resolution of inflammatory pain. J Clin Invest 128: 3568-3582, 2018.

19. Xu Q, Du F, Zhang Y, Teng Y, Tao M, Chen AF and Jiang R: Preeclampsia serum induces human glomerular vascular endothelial cell hyperpermeability via the HMGB1-Caveolin-1 pathway. J Reprod Immunol 129: 1-8, 2018

20. Zhang X, Ramírez CM, Aryal B, Madrigal-Matute J, Liu X, Diaz A, Torrecilla-Parra M, Suárez Y, Cuervo AM, Sessa WC and Fernández-Hernando C: Cav-1 (Caveolin-1) deficiency increases autophagy in the endothelium and attenuates vascular inflammation and atherosclerosis. Arterioscler Thromb Vasc Biol 40: 1510-1522, 2020

21. Chung JW, Kim DH, Oh MJ, Cho YH, Kim EH, Moon GJ, Ki CS Cha J, Kim KH, Jeon P, et al: Cav-1 (Caveolin-1) and arterial remodeling in adult moyamoya disease. Stroke 49: 2597-2604, 2018.

22. Flatters SJ: Characterization of a model of persistent postoperative pain evoked by skin/muscle incision and retraction (SMIR) Pain 135: 119-130, 2008.

23. Zahn PK, Pogatzki EM and Brennan TJ: Mechanisms for pain caused by incisions. Reg Anesth Pain Med 27: 514-516, 2002

24. Livak KJ and Schmittgen TD: Analysis of relative gene expression data using real-time quantitative PCR and the 2(-Delta Delta C(T)) method. Methods 25: 402-408, 2001.

25. Mirra A, Spadavecchia C, Bruckmaier R, Gutzwiller A and Casoni D: Acute pain and peripheral sensitization following cautery disbudding in 1- and 4-week-old calves. Physiol Behav 84: 248-260, 2018.

26. Zhang Y and Wang Y: TRPV1: An important molecule involved in the peripheral sensitization during chronic pain and central pain modulation. Sheng Li Xue Bao 25: 677-684, 2017 (In Chinese).

27. Filippini A and D'Alessio A: Caveolae and lipid rafts in endothelium: Valuable organelles for multiple functions. Biomolecules 10: 1218, 2020 .

28. Chow BW, Nuñez V, Kaplan L, Granger AJ, Bistrong K, Zucker HL, Kumar P, Sabatini BL and Gu C: Caveolae in CNS arterioles mediate neurovascular coupling. Nature 579: 106-110, 2020

29. Jin $\mathrm{H}, \mathrm{Xu} \mathrm{Y}$, Shi F and $\mathrm{Hu} \mathrm{S}$ : Vaccination at different anatomic sites induces different levels of the immune responses. Res Vet Sci 122: 50-55, 2019.

30. Parton RG, Tillu VA and Collins BM: Caveolae. Curr Biol 23 : R402-R405, 2018.
31. Oliveira SDS, Chen J, Castellon M, Mao M, Raj JU, Comhair S, Erzurum S, Silva CLM, Machado RF, Bonini MG and Minshall RD: Injury-induced shedding of extracellular vesicles depletes endothelial cells of Cav-1 (Caveolin-1) and enables TGF- $\beta$ (Transforming Growth Factor- $\beta$ )-dependent pulmonary arterial hypertension. Arterioscler Thromb Vasc Biol 39: 1191-1202, 2019.

32. Luo M, Xu C, Luo Y, Wang G, Wu J and Wan Q: Circulating miR-103 family as potential biomarkers for type 2 diabetes through targeting CAV-1 and SFRP4. Acta Diabetol 57: 309-322, 2020.

33. Wang DX, Pan YQ, Liu B and Dai L: Cav-1 promotes atherosclerosis by activating JNK-associated signaling. Biochem Biophys Res Commun 503: 513-520, 2018.

34. Konrad FM, Meichssner N, Bury A, Ngamsri KC and Reutershan J: Inhibition of SDF-1 receptors CXCR4 and CXCR7 attenuates acute pulmonary inflammation via the adenosine A2B-receptor on blood cells. Cell Death Dis 8: e2832, 2017.

35. Wang N, Zhang D, Sun G, Zhang H, You Q, Shao M and Yue Y: Lipopolysaccharide-induced caveolin-1 phosphorylation-dependent increase in transcellular permeability precedes the increase in paracellular permeability. Drug Des Devel Ther 9: 4965-4977, 2015.

36. Andreone BJ, Chow BW, Tata A, Lacoste B, Ben-Zvi A, Bullock K, Deik AA, Ginty DD, Clish CB and Gu C: Blood-brain barrier permeability is regulated by lipid transport-dependent suppression of caveolae-mediated transcytosis. Neuron 94: 581-594, 2017.

37. Bravo-Caparrós I, Ruiz-Cantero MC, Perazzoli G, Cronin SJF, Vela JM, Hamed MF, Penninger JM, Baeyens JM, Cobos EJ and Nieto FR: Sigma-1 receptors control neuropathic pain and macrophage infiltration into the dorsal root ganglion after peripheral nerve injury. FASEB J 34: 5951-5966, 2020.

38. Mekhail N, Deer TR, Poree L, Staats PS, Burton AW, Connolly AT, Karst E, Mehanny DS, Saweris Y and Levy RM: Cost-effectiveness of dorsal root ganglion stimulation or spinal cord stimulation for complex regional pain syndrome. Neuromodulation 9: 708-714, 2020.

39. He DD, Gao Y, Wang S, Xie Z and Song XJ: Systematic administration of $\mathrm{B}$ vitamins alleviates diabetic pain and inhibits associated expression of P2X3 and TRPV1 in dorsal root ganglion neurons and proinflammatory cytokines in spinal cord in rats. Pain Res Manag 10: 3740162, 2020

40. Li QY, Xu HY and Yang HJ: Effect of proinflammatory factors TNF- $\alpha, I L-1 \beta$, IL- 6 on neuropathic pain. Zhongguo Zhong Yao Za Zhi 42: 3709-3712, 2017 (In Chinese)

41. Ding HH, Zhang SB, Lv YY, Ma C, Liu M, Zhang KB, Ruan XC, Wei JY, Xin WJ and Wu SL: TNF- $\alpha$ /STAT3 pathway epigenetically upregulates Nav1.6 expression in DRG and contributes to neuropathic pain induced by L5-VRT. J Neuroinflammation 16: 29, 2019.

42. Zhang ZJ, Jiang BC and Gao YJ: Chemokines in neuron-glial cell interaction and pathogenesis of neuropathic pain. Cell Mol Life Sci 74: 3275-3291, 2017.

43. Li CD, Zhao JY, Chen JL, Lu JH, Zhang MB, Huang Q, Cao YN, Jia GL, Tao YX, Li J and Cao H: Mechanism of the JAK2/STAT3-CAV-1-NR2B signaling pathway in painful diabetic neuropathy. Endocrine 64: 55-66, 2019.

44. Kassan A, Egawa J, Zhang Z, Almenar-Queralt A, Nguyen QM, Lajevardi Y, Kim K, Posadas E, Jeste DV, Roth DM, et al: Caveolin-1 regulation of disrupted-in-schizophrenia-1 as a potential therapeutic target for schizophrenia. J Neurophysiol 117: 436-444, 2017

45. Sheikh NK and Dua A: Neuroanatomy, substantia gelatinosa. In: StatPearls. StatPearls Publishing, Treasure Island, FL, 2020.

46. Roh J, Hwang SM, Lee SH, Lee K, Kim YH and Park CK: Functional expression of piezol in dorsal root ganglion (DRG) neurons. Int J Mol Sci 21: 3834, 2020.

47. Zhang J, Harada Y and Hayashi Y: A TLR-CXCL1 pathway in DRG neurons induces neutrophil accumulation in the DRG and mechanical allodynia in EAE mice. Sci Rep 9: 12003, 2019.

48. Huang $H$, Wang $M$ and Hong Y: Intrathecal administration of adrenomedullin induces mechanical allodynia and neurochemical changes in spinal cord and DRG. Neurosci Lett 690: 196-201, 2019.

This work is licensed under a Creative Commons Attribution-NonCommercial-NoDerivatives 4.0 International (CC BY-NC-ND 4.0) License. 